\title{
A matched-control comparison of serious adverse events after intravitreal injections of bevacizumab for age-related macular degeneration and cataract extraction
}

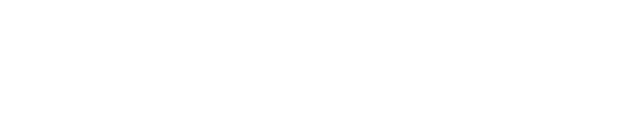

\section{Naomi Fischer \\ Elad Moisseiev \\ Michael Waisbourd \\ Michaella Goldstein \\ Anat Loewenstein}

Department of Ophthalmology, Tel Aviv Medical Center, Sackler Faculty of Medicine, Tel Aviv University, Tel Aviv, Israel
Correspondence: Elad Moisseiev Department of Ophthalmology, Tel Aviv Sourasky Medical Center, Weitzman $6 \mathrm{St}$, Tel Aviv, 64239 Israel

Tel +97236973408

Fax +97236973870

Email elad_moi@netvision.net.il
Purpose: The study reported here investigated the rates of systemic serious adverse events (SAEs) following treatment with intravitreal bevacizumab for age-related macular degeneration (AMD) in comparison with a matched control group.

Methods: A retrospective age- and sex-matched case-control design was used. Data were collected using patient charts and telephone surveys. The main outcome measure was difference in number of hospital admissions between the two groups. Hospitalizations were further analyzed according to whether or not they were due to arteriothrombotic SAEs.

Results: Each group comprised 65 participants. There were significantly more hospital admissions among bevacizumab-treated patients than in the control group $(P=0.039)$. Sub-analysis of hospitalizations due to arteriothrombotic causes did not reveal a statistically significant difference between groups $(P=0.629)$.

Conclusion: The results suggest that intravitreal bevacizumab is not associated with an increased risk of arteriothrombotic SAEs. Its widespread use for the treatment of AMD appears to be systemically safe.

Keywords: Avastin ${ }^{\circledR}$, serious adverse events, safety

\section{Introduction}

Age-related macular degeneration (AMD) is the leading cause of irreversible severe central visual acuity loss in people over 50 years of age in the developed world. ${ }^{1}$ The disease affects more than $30 \%$ of the population over 75 years of age. ${ }^{2}$ Approximately $20 \%$ of patients with AMD advance to the exudative stage and develop choroidal neovascularization, which is responsible for almost $90 \%$ of severe vision loss in this disease. . $^{3,4}$

The current mainstay treatment for exudative AMD is intravitreal injections of anti-vascular endothelial growth factor (anti-VEGF) agents. Large-scale, prospective, randomized, controlled multicenter studies have established the efficacy of ranibizumab (Lucentis ${ }^{\circledR}$, Genentech, San Francisco, CA, USA) in the treatment of neovascular AMD. ${ }^{5,6}$ Ranibizumab is a monoclonal antibody fragment derived from bevacizumab (Avastin ${ }^{\circledR}$, Genentech/Roche, Basel, Switzerland), a humanized monoclonal antibody that inhibits VEGF-A and was first approved by the US Food and Drug Administration in 2004 for metastatic colon cancer. ${ }^{7}$ Ranibizumab has a stronger binding affinity to VEGF-A but a shorted half-life in animal models, ${ }^{8,9}$ and has been approved by the US Food and Drug Administration for the treatment of neovascular AMD. However, bevacizumab 
has been extensively and effectively used off-label for this indication, mostly for economic reasons. ${ }^{10}$ Recently, the results of two large-scale, multicenter, randomized, controlled comparative studies have demonstrated that bevacizumab is non-inferior to ranibizumab in its effect on visual acuity when administered according to the same regimen. ${ }^{11,12}$ These results support the use of bevacizumab, and it is therefore expected to be used more frequently in the future.

Systemic intravenous treatment with bevacizumab in patients with metastatic cancer is associated with an increased risk of thromboembolic events. ${ }^{7,13,14}$ Ranibizumab is only used intravitreally. Both drugs are associated with ocular adverse events, which are very rare and may be related to complications of the injection rather than the specific agents. These ocular adverse events include endophthalmitis, intraocular inflammation, increased intraocular pressure, vitreous hemorrhage, retinal detachment, retinal pigment epithelial tears, and cataract. $5,6,15,16$

The dose of bevacizumab in intravitreal injections is approximately 420 to 500 times smaller than that used systemically. It has been theorized that its systemic penetration from the vitreous cavity is limited by the blood-ocular barrier, although this may be compromised by choroidal neovascularization. ${ }^{17,18}$ Several studies have shown that intravitreal injection of bevacizumab has greater systemic penetrance and effect than ranibizumab..$^{8,9,19,20}$ It has also been shown that systemic levels of VEGF are lower in patients treated with bevacizumab than in those treated with ranibizumab. ${ }^{12,21-23}$ The aforementioned comparative studies of the efficacy of these two agents in the treatment of AMD were insufficiently powered to detect differences in SAEs between them. ${ }^{11,12}$

However, although unproven, there is general concern among practicing ophthalmologists that treatment with bevacizumab may be associated with a higher risk of SAEs, especially arteriothrombotic SAEs, due to its greater systemic penetrance. Therefore, the purpose of the study reported here was to investigate whether there is an increase in systemic SAEs following treatment with intravitreal bevacizumab for AMD in comparison with a matched control group.

\section{Materials, methods, and subjects}

Patients included in this study were recruited from the database listing patients who underwent intravitreal injections or cataract extraction procedures in our department between January 1, 2006 and December 31, 2008.

The study group included patients who received their first intravitreal injection of bevacizumab during this period for the treatment of exudative AMD. The medical records of these patients were retrospectively reviewed, and patients were excluded if they were treated for any indication other than AMD, had also been treated by intravitreal ranibizumab or systemic bevacizumab, or if they had less than 12 months of follow-up.

The control group was designed to match the study group in terms of sex and age. This group included patients who underwent cataract extraction during the same period but who did not receive any anti-VEGF-agent treatment.

Data were retrieved from outpatient clinic charts, hospital admission and discharge letters, and a telephone questionnaire focused on hospitalizations and SAEs that occurred during the follow-up period (Table 1). Recorded parameters for each patient included demographic information, number of bevacizumab injections, background illnesses and medical conditions, and the occurrence of any hospitalizations during the follow-up period. Follow-up time was calculated as the period between the bevacizumab injection or cataract extraction and the time of completion of the questionnaire.

The main outcome measure of this study was hospital admissions. Hospitalizations were further categorized according to their reason being arteriothrombotic or not. Arteriothrombotic reasons included myocardial infarctions,

Table I Telephone questionnaire

Question I: When was your first intravitreal Avastin/cataract surgery injection and in which eye?

Question 2: Have you been hospitalized since the first injection until today? If yes:

A. When?

B. In which hospital were you hospitalized?

C. What was the reason for hospitalization?

D. How much time had passed from the injection until hospitalization?

Question 3: Do you suffer from any one of the following conditions?

A. Ischemic heart disease (angina pectoris, myocardial infarction, heart catheterization, coronary artery bypass graft surgery)

B. Diabetes mellitus (if yes, for how many years?)

C. Hypertension

D. Hyperlipidemia

E. Impaired renal function or chronic renal function (CRF)

F. Vascular disease (carotid artery stenosis or occlusion, carotid endarterectomy, peripheral vascular disease [PVD])

G. Cerebral vascular accident (CVA) or transient ischemic attack (TIA) $\mathrm{H}$. Venous thrombotic event

If yes, was it/were they present prior to the injection/cataract surgery or diagnosed after it?

Question 4: Do you currently smoke?*

Question 5: Are you currently overweight?*

Question 6: Are you physically active (at least three times a week)?*

Notes: *These questions asked with regard to the time of the questionnaire. Answers to questions were categorized qualitatively (yes/no) and no quantitative evaluation of these parameters was recorded. 
cerebrovascular accidents, transient ischemic attack, or venous thrombosis.

The institutional review board of our institution approved the study, and all patients received a verbal explanation of the study and verbally expressed their consent to participate prior to completing the telephone questionnaire.

Correlations between continuous variables were analyzed using Pearson's correlation coefficient, and Chi-square tests were used to analyze associations between categorical parameters. Data were analyzed using SPSS for Windows (v 17.0; IBM, Armonk, NY, USA). A $P$ value of 0.05 was used to declare statistically significant difference between groups.

\section{Results}

Ninety patients who received intravitreal injections of bevacizumab were randomly selected from the database list. Of these, 65 patients fulfilled the inclusion criteria and were included in the study. A control group of 65 sex- and age-matched patients was selected from the database list of patients who underwent cataract extraction.

Both groups included 24 men (36.9\%) and 41 women $(63.1 \%)$. Mean age was $80.43 \pm 7.61$ years in the bevacizumab-treated group and $80.28 \pm 7.49$ years in the control group $(P=0.908)$. Mean follow-up was $24.0 \pm 5.6$ months for bevacizumab-treated patients and $26.3 \pm 2.8$ months for the controls $(P=0.766)$. Throughout the follow-up, bevacizumab-treated patients underwent a mean of $5.1 \pm 4.1$ injections (range 1-20). There were no statistically significant differences in any background medical conditions between the patients in both groups, except for a higher rate of chronic renal failure in the control group (Table 2). No deaths were encountered in this study.

Table 2 Comparison of the background medical conditions of patients in both groups

\begin{tabular}{llll}
\hline $\begin{array}{l}\text { Background } \\
\text { condition }\end{array}$ & $\begin{array}{l}\text { Bevacizumab- } \\
\text { treated group, } \\
\text { n (\%) }\end{array}$ & $\begin{array}{l}\text { Control } \\
\text { group, } \\
\text { n (\%) }\end{array}$ & P \\
\hline Diabetes mellitus & $15(23)$ & $23(35.3)$ & $>0.05$ \\
Hypertension & $4 I(63)$ & $4 I(63)$ & $>0.05$ \\
Ischemic heart disease & $8(12.3)$ & $13(20)$ & $>0.05$ \\
Vascular disease & $3(4.6)$ & $2(3)$ & $>0.05$ \\
Hyperlipidemia & $32(49.2)$ & $38(58.4)$ & $>0.05$ \\
Chronic renal failure & $3(4.6)$ & $11(16.9)$ & 0.043 \\
Cancer & $7(10.7)$ & $6(9.2)$ & $>0.05$ \\
Smoking & $11(16.9)$ & $7(10.7)$ & $>0.05$ \\
Overweight & $15(23)$ & $12(18.4)$ & $>0.05$ \\
Physical activity & $29(44.6)$ & $20(30.7)$ & $>0.05$ \\
\hline
\end{tabular}

Overall, there were more hospital admissions in the bevacizumab-treated group. During the follow-up period, there were 21 hospital admissions among the 65 patients $(32.3 \%)$ in the study group compared with ten hospital admissions among the control patients (15.3\%). This difference was statistically significant $(P=0.039)$. However, further analysis revealed that only a minority of these hospital admissions was due to arteriothrombotic events. Arteriothrombotic events were the reason for the hospitalizations of three $(4.6 \%)$ patients who were treated with bevacizumab and one patient (1.5\%) from the control group. This analysis was not found to be statistically significant $(P=0.629)$.

\section{Discussion}

The results of this study indicate that patients treated with bevacizumab had more systemic SAEs than their age- and sex-matched counterparts in the control group. Arteriothrombotic SAEs were slightly more common among patients treated with bevacizumab, but this difference was not statistically significant. As far as the authors are aware, this is the first study to have compared the rate of systemic SAEs of intravitreal bevacizumab with a matched control group and for a period of over 2 years of follow-up.

In the Comparison of AMD Treatments Trials (CATT), patients treated with bevacizumab had more systemic SAEs than with ranibizumab. However, the majority of these SAEs were hospitalizations, mostly for reasons unrelated to the antiVEGF therapy, and the proportions of patients who suffered arteriothrombotic SAEs were similar for both drugs. ${ }^{11}$ In the alternative treatments to inhibit VEGF in age-related choroidal neovascularisation (IVAN), both drugs were associated with similar rates of SAEs, and arteriothrombotic events were less frequent among patients treated with bevacizumab. ${ }^{12}$ A meta-analysis by van der Reis et al revealed that, overall, systemic SAEs were lower for intravitreal bevacizumab than for ranibizumab and pegaptanib. ${ }^{24}$ Overall, it was concluded that there is no sufficient evidence to prove there is a difference in rates of adverse events between these anti-VEGF drugs.

A comparative study of the systemic SAEs associated with intravitreal bevacizumab would require a very large cohort, and this has not been undertaken. Systemic bevacizumab has been associated with arteriothrombotic complications, ${ }^{7,13,14}$ and intravitreal bevacizumab has been shown to have systemic penetration. ${ }^{8,9,19,20}$ However, despite these facts, its intravitreal administration has not been associated with arteriothrombotic SAEs. The findings of our study are in accordance with the data accumulated in previous studies and suggest that the risk for arteriothrombotic SAEs associated with bevacizumab therapy for AMD is low. 
It should be noted that the overall rate of hospitalizations was higher among patients treated with bevacizumab in our study. This finding is similar to that of the CATT study, ${ }^{11}$ but its clinical significance is unclear, since most of the hospitalizations were due to reasons unrelated to the anti-VEGF therapy. It should also be noted that an association between cardiovascular disease and AMD has been suggested, ${ }^{25,26}$ and this may serve as a confounding factor for the risk of thromboembolic events after intravitreal treatment with bevacizumab. Further, the rare occurrence of some SAEs may simply be coincidental. ${ }^{24}$

\section{Limitations}

A limitation of this study is its relatively small size. Although previous larger studies were also underpowered to evaluate SAEs associated with intravitreal bevacizumab, this study is unique in its design, and evaluated this important issue from a new perspective. Another limitation is the use of a questionnaire, which may have led to a recall bias.

\section{Conclusion}

Our results suggest a low risk for arteriothrombotic SAEs with administration of intravitreal bevacizumab for AMD. Although hospitalizations were more common in these patients, the clinical importance of this finding is unclear. Multicenter, randomized, controlled studies powered to investigate systemic SAEs of intravitreal bevacizumab as well as ranibizumab need to be initiated in the future. In the meantime, we suggest that the use of intravitreal bevacizumab appears to be systemically safe.

\section{Disclosure}

None of the authors has any financial or proprietary interests in this study and no conflicting relationship exists for any author in relation to this work. No grants or funds were received to finance this study.

\section{References}

1. Bressler NM. Age-related macular degeneration is the leading cause of blindness. JAMA. 2004;291(15):1900-1901.

2. Klein R, Klein BE, Linton KL. Prevalence of age-related maculopathy. The Beaver Dam Eye Study. Ophthalmology. 1992;99(6):933-943.

3. Kahn HA, Leibowitz HM, Ganley JP, et al. The Framingham Eye Study. I. Outline and major prevalence findings. Am J Epidemiol. 1977;106(1):17-32.

4. Ferris FL 3rd, Fine SL, Hyman L. Age-related macular degeneration and blindness due to neovascular maculopathy. Arch Ophthalmol. 1984;102(11):1640-1642.

5. Rosenfeld PJ, Brown DM, Heier JS, et al; MARINA Study Group. Ranibizumab for neovascular age-related macular degeneration. $N$ Engl J Med. 2006;355(14):1419-1431.
6. Brown DM, Kaiser PK, Michels M, et al; ANCHOR Study Group. Ranibizumab versus verteporfin for neovascular age-related macular degeneration. N Engl J Med. 2006;355(14):1432-1444.

7. Genentech. Avastin ${ }^{\circledR}$ (bevacizumb) [product characteristics]. San Francisco, CA: Genentech; 2013. Available from: http://www.gene. com/gene/products/information/pdf/avastin-prescribing.pdf. Accessed March 3, 2013.

8. Bakri SJ, Snyder MR, Reid JM, Pulido JS, Singh RJ. Pharmacokinetics of intravitreal bevacizumab (Avastin). Ophthalmology. 2007; 114(5):855-859.

9. Bakri SJ, Snyder MR, Reid JM, Pulido JS, Ezzat MK, Singh RJ. Pharmacokinetics of intravitreal ranibizumab (Lucentis). Ophthalmology. 2007;114(12):2179-2182.

10. Ciulla TA, Rosenfeld PJ. Anti-vascular endothelial growth factor therapy for neovascular ocular diseases other than age-related macular degeneration. Curr Opin Ophthalmol. 2009;20(3):166-174.

11. CATT Research Group, Martin DF, Maguire MG, et al. Ranibizumab and bevacizumab for neovascular age-related macular degeneration. N Engl J Med. 2011;364(20):1897-1908.

12. IVAN Study Investigators, Chakravarthy U, Harding SP, et al. Ranibizumab versus bevacizumab to treat neovascular age-related macular degeneration: one-year findings from the IVAN randomized trial. Ophthalmology. 2012;119(7):1399-1411.

13. Nalluri SR, Chu D, Keresztes R, Zhu X, Wu S. Risk of venous thromboembolism with the angiogenesis inhibitor bevacizumab in cancer patients: a meta-analysis. JAMA. 2008;300(19):2277-2285.

14. Scappaticci FA, Skillings JR, Holden SN, et al. Arterial thromboembolic events in patients with metastatic carcinoma treated with chemotherapy and bevacizumab. J Natl Cancer Inst. 2007;99(16):1232-1239.

15. Wong LJ, Desai RU, Jain A, et al. Surveillance for potential adverse events associated with the use of intravitreal bevacizumab for retinal and choroidal vascular disease. Retina. 2008;28(8):1151-1158.

16. Georgopoulos M, Polak K, Prager F, Prünte C, Schmidt-Erfurth U. Characteristics of severe intraocular inflammation following intravitreal injection of bevacizumab (Avastin). Br J Ophthalmol. 2009; 93(4):457-462.

17. Gariano RF, Gardner TW. Retinal angiogenesis in development and disease. Nature. 2005;438(7070):960-966.

18. van Wijngaarden P, Coster DJ, Williams KA. Inhibitors of ocular neovascularization: promises and potential problems. JAMA. 2005; 293(12):1509-1513.

19. Byeon SH, Kang SY. Pharmacokinetics. Ophthalmology. 2009; 116(1):168-169.

20. Meyer CH, Holz FG. Preclinical aspects of anti-VEGF agents for the treatment of wet AMD: ranibizumab and bevacizumab. Eye (Lond). 2011;25(6):661-672.

21. Davidović SP, Nikolić SV, Curić NJ, et al. Changes of serum VEGF concentration after intravitreal injection of Avastin in treatment of diabetic retinopathy. Eur J Ophthalmol. 2012;22(5):792-798.

22. Sato T, Wada K, Arahori H, et al. Serum concentrations of bevacizumab (avastin) and vascular endothelial growth factor in infants with retinopathy of prematurity. Am J Ophthalmol. 2012; 153(2):327-333. e1.

23. Avery RL, Pearlman J, Pieramici DJ, et al. Intravitreal bevacizumab (Avastin) in the treatment of proliferative diabetic retinopathy. Ophthalmology. 2006;113(10):1695. e1-e15.

24. van der Reis MI, La Heij EC, De Jong-Hesse Y, Ringens PJ, Hendrikse F, Schouten JS. A systematic review of the adverse events of intravitreal anti-vascular endothelial growth factor injections. Retina. 2011;31(8):1449-1469.

25. Tan JS, Mitchell P, Smith W, Wang JJ. Cardiovascular risk factors and the long-term incidence of age-related macular degeneration: the Blue Mountains Eye Study. Ophthalmology. 2007;114(6):1143-1150.

26. Tan JS, Wang JJ, Liew G, Rochtchina E, Mitchell P. Age-related macular degeneration and mortality from cardiovascular disease or stroke. $\mathrm{Br} J$ Ophthalmol. 2008;92(4):509-512. 
Clinical Ophthalmology

\section{Publish your work in this journal}

Clinical Ophthalmology is an international, peer-reviewed journal covering all subspecialties within ophthalmology. Key topics include: Optometry; Visual science; Pharmacology and drug therapy in eye diseases; Basic Sciences; Primary and Secondary eye care; Patien Safety and Quality of Care Improvements. This journal is indexed on

PubMed Central and CAS, and is the official journal of The Society of Clinical Ophthalmology (SCO). The manuscript management system is completely online and includes a very quick and fair peer-review system, which is all easy to use. Visit http://www.dovepress.com/ testimonials.php to read real quotes from published authors. 